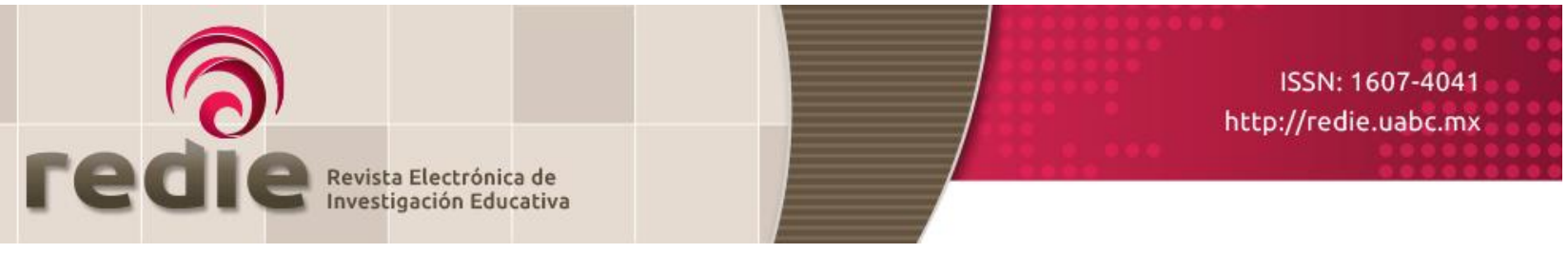

Vol. 20, Núm. 2, 2018

\title{
Escala de diagnóstico de las relaciones familia-escuela: análisis psicométrico
}

\section{Diagnosis Scale for Family-School Relationships: a Psychometric Analysis}

Antonio Ramírez-García (1) ed1ragaa@uco.es

Victoriano Anguita-López (2) anlov@uco.es

José Carlos Casas del Rosal (1) jcasas@uco.es

(1) Universidad de Córdova

(2) Servicio de Inspección Educativa de Córdova

(Recibido: 15 de agosto de 2016; Aceptado para su publicación: 24 de noviembre de 2016)

Cómo citar: Ramírez-García, A., Anguita-López, V. y Casas, J. C. (2018). Escala de diagnóstico de las relaciones familia-escuela: análisis psicométrico. Revista Electrónica de Investigación Educativa, 20(2), 69-79. https://doi.org/10.24320/redie.2017.20.2.1529

\section{Resumen}

Familia y Escuela son los dos principales agentes socializadores responsables de la educación de los niños. Con el objeto de profundizar en el conocimiento de ambas instituciones y las necesarias relaciones entre éstas se propone la Escala de Diagnóstico de las Relaciones Familia-Escuela (DIREFAES); en el presente estudio se hace un análisis psicométrico de la escala en una muestra de 1,276 familias cordobesas (España). El análisis estadístico de los ítems constata su poder discriminante y el análisis factorial demuestra la validez teórica de los componentes. Se identifican 13 factores coincidentes en un alto grado con las ocho dimensiones resultantes del análisis teórico y normativo y la participación de un grupo de expertos. Por último, a través del empleo del alfa de Cronbach se confirma la fiabilidad de la herramienta tanto en su conjunto (.951) como en las diferentes dimensiones (excepto una, el resto es superior a .817).

Palabras clave: Familia, escuela, análisis psicométrico.

\section{Abstract}

Family and school are the two main socializing agents responsible for children's education. In order to gain greater insight into both institutions and the necessary links between them, this study proposes the Diagnosis Scale for Family-School Relationships (DIREFAES, in Spanish) and conducts a psychometric analysis of the scale in a sample of 1,276 families from Córdoba, Spain. A statistical analysis of items confirms its discriminatory power and a factorial analysis establishes the theoretical validity of components. The study identified 13 factors that coincide to a high degree with the eight dimensions resulting from a theoretical and policy analysis and the participation of a group of experts. Finally, Cronbach's alpha was used to confirm the reliability of the tool both as a whole (.951) and in its various dimensions (except for one; the rest were above .817). 


\section{Introducción}

El modelo social que se ha venido configurando en las dos primeras décadas del siglo XXI es fruto, según Fraguela, Lorenzo y Varela (2011), de las transformaciones que han tenido lugar en la estructura familiar (García, Gomariz, Hernández y Parra, 2010). Este contexto familiar también repercute en la institución educativa que, a su vez, se ha visto sometida a sus propios procesos de transformación (García et al., 2010).

Рara Gamarra y Carrasco (2013), la familia y la escuela se configuran como las primeras instancias de aprendizaje, dos contextos diferenciados que están obligados a mantener una estrecha interconexión. Diferentes estudios han revelado la importancia de esta colaboración, ya que implica un importante factor de integración y cohesión social (Santos, Lorenzo y Priegue, 2011), posibilita la inclusión del alumnado en el centro (Lozano, Alcaraz y Colás, 2013), genera el incremento del rendimiento escolar (Bolívar, 2006; Chiu y Xihua, 2008; Dearing, Kreider y Weiss, 2006; Driessen, Smit y Sleegers, 2005; OECD, 2012; Ruiz de Miguel, 2009; Villiger, Niggli y Wandeler, 2011), favorece el aumento de la motivación y su repercusión en los resultados (Córdoba, García, Luengo, Vizuete y Feu, 2011; Fan, Williams y Wolters, 2011; Fraguela, Lorenzo y Varela, 2011), permite la creación de actitudes y estados de motivación idóneos para afrontar el aprendizaje (Bodovski y Joun, 2010; Rodríguez, Drognett y Revuelta, 2012) y consigue un aumento de las expectativas recíprocas respecto a la educación (Sarramona y Roca, 2007).

Junto a este contexto de acción se ha de considerar la evolución de la psicometría en la medición de esta realidad. En este sentido, cabría mencionar, sobre todo, el desarrollo de la Teoría de Respuesta al ítem (IRT, por sus siglas en inglés); Lord, 1980; Van der Linden y Hambleton, 1997; Hambleton, Swaminathan y Rogers, 1991), caracterizada por ser una aproximación a la medida basada en modelos y por disponer de técnicas para evaluar el ajuste del modelo, que puede realizarse en tres niveles: 1) valorando el ajuste entre valores observados y esperados bajo el modelo estimado, mediante el uso de pruebas de bondad de ajuste referidas a los ítems (ji-cuadrado, análisis de residuales y razón de verosimilitud) y pruebas de bondad de ajuste del modelo sustentadas en la comparación entre modelos, 2) evaluando los supuestos del modelo (dimensionalidad, independencia local, igualdad de los parámetros de discriminación, ausencia de adivinación y ausencia de velocidad), y 3) evaluando si se cumplen las ventajas derivadas del modelo. Asimismo, posibilita el marco para analizar el Funcionamiento Diferencial del ítem (DIF, por sus siglas en inglés) y el Funcionamiento Diferencial del Test (DTF, por sus siglas en inglés), entre otras cuestiones.

En la aplicación de la IRT es necesario optar por un modelo que permita conocer el rendimiento en los ítems, los más utilizados son los logísticos de uno, dos o tres parámetros, destacando el Modelo de Rasch.

De forma concreta, las investigaciones que se han centrado en medir determinados aspectos de las relaciones familia y escuela utilizan modelos lineales generales de análisis multivariante de la covarianza (Pepe y Addimando, 2014), el análisis factorial para medir las propiedades psicométricas de un cuestionario sobre experiencia de padres con escuelas internacionales (Patterson, 2016), el análisis de la confiabilidad de los puntajes mediante la Teoría de Respuesta al ítem (el modelo de crédito parcial, adaptación del modelo de Rasch para ítems politómicos), el análisis factorial exploratorio con el método de máxima verosimilitud y rotación Oblimin, y el modelamiento estructural mediante el método de estimación Distribución Libre Asintótica (ADI) para medir la participación de los padres en la educación escolar de sus hijos (Valdés, Carlos y Arreola, 2013), los modelos politómicos de Rasch (Modelo de Crédito Parcial y Modelo de Escalas de Clasificación, entre otros) (Navarro, Expósito, López y Thoilliez, 2014) para la validación de una escala de percepción de indicadores de bienestar infantil en la que se incluyen dimensiones como el bienestar material de las familias y las relaciones con el entorno (familia, escuela, comunidad, etc.) o el Modelo de Rasch para conformar y calibrar las escalas de aprendizaje y las de contexto (dinámica familiar y capital cultural, entre otras), el Modelo de Ecuaciones Estructurales para validar dichas escalas y los Modelos Jerárquicos Lineales para identificar y cuantificar las relaciones existentes entre las distintas variables de contexto con el logro educativo (Backhoff et al. 2008), entre otros. 
Todas estas referencias contribuyen a comprender mejor las variables que intervienen y condicionan las relaciones entre familia y escuela, consideradas como un indicador de calidad de los sistemas educativos (Colás y Contreras, 2013), al tiempo que son prescriptivas en el funcionamiento de los centros escolares (Domingo, Martos y Domingo, 2010) y requieren de una regulación en cuanto a formas de participación, fórmulas de colaboración o instrumentos empleados en la comunicación, entre otras cuestiones. Así, partiendo de ello, a nivel teórico se establecieron las 8 dimensiones que constituirían el constructo de dichas relaciones: conocimiento de las familias sobre aspectos del centro, recursos utilizados para la comunicación entre la escuela y la familia, contenidos de las comunicaciones con las familias, participación de las familias en actividades del centro, participación de las familias en estructuras organizativas del centro, contribución de las familias en el aprendizaje de sus hijos e hijas, facilidades para la colaboración/participación en el centro escolar y compromisos de mejora por parte de las familias. En este sentido, el objetivo de este trabajo es realizar un análisis psicométrico para determinar la fiabilidad y validez de un instrumento que posibilita establecer un diagnóstico de las relaciones entre la familia y la escuela.

\section{Método}

El procedimiento se realizó en dos fases: 1) Diseño y validación de la escala, y 2) Identificación de las propiedades psicométricas del instrumento.

El diseño de la escala (DIREFAES) parte del cuestionario de García et al. (2010) y del análisis de la normativa vigente andaluza en relación con la orientación y acción tutorial en los centros educativos, ${ }^{1}$ especialmente aspectos vinculados a la relación familia-escuela en la etapa de educación primaria (6-12 años). El procedimiento llevado a cabo para el diseño y validación del cuestionario siguió las indicaciones de la American Educational Research Association, la American Psychological Association y el National Council for Measurement in Education (AERA, APA y NCME, 1999), Carretero-Dios y Pérez (2005), Martínez-Arias (1995) y Muñiz (1998). Tras la elaboración provisional del cuestionario se empleó el método Delphi para comprobar la validez de contenido del mismo. El grupo de expertos estuvo constituido por 6 profesores universitarios, 3 orientadores y 2 maestros-tutores de educación primaria, cumpliendo así los requisitos postulados por Pozo, Gutiérrez y Rodríguez (2007). Se diseñó una plantilla de respuesta formada por 15 ítems, de los cuales, tres eran de respuesta abierta, para estimar la idoneidad, estructura, adecuación, claridad y calidad de los ítems de la herramienta. En esta plantilla se solicitó al grupo de expertos su valoración sobre la idoneidad de las ocho dimensiones, así como la claridad en la formulación de los ítems y su relevancia dentro de la dimensión en la que está incluida. Así, la V de Aiken arrojó un valor de 0.92 para el conjunto de la escala. La valoración global del grupo de expertos, escogiendo como criterio que las valoraciones coincidieran en el $70 \%$ de los casos, permitió reducir de 74 a 66 los ítems del cuestionario (valoración escalar tipo Likert de 1 a 5), se matizó el vocabulario utilizado en la redacción de los mismos y se ejemplificaron algunos ítems para su comprensión. Como resultado, el constructo se configuró en torno a las ocho dimensiones mencionadas anteriormente.

Tras la redacción final del cuestionario se realizó la encuesta a los padres de familia en diversos centros de educación infantil y primaria situados tanto en Córdoba capital como en zonas rurales de la provincia, de

\footnotetext{
${ }^{1}$ Decreto 328/2010, de 13 de Julio por el que se aprueba el reglamento orgánico de las escuelas infantiles de segundo ciclo, de los colegios de Educación Primaria, de los colegios de educación infantil y primaria y de los centros públicos específicos de educación especial (Boletín Oficial de la Junta de Andalucía, número 139, de 16 de julio de 2010); Orden de 20 de agosto de 2010, por la que se regula la organización y el funcionamiento de las escuelas infantiles de segundo ciclo, de los colegios de Educación Primaria, de los colegios de educación infantil y primaria y de los centros públicos específicos de educación especial, así como el horario de los centros, del alumnado y del profesorado (Boletín Oficial de la Junta de Andalucía, número 204, del 19 de octubre de 2005); Orden del 20 de junio de 2011, por la que se adoptan medidas para la promoción de la convivencia en los centros docentes sostenidos con fondos públicos y se regula el derecho de las familias a participar en el proceso educativo de sus hijos e hijas. (Boletín Oficial de la Junta de Andalucía, número 132, de 7 de julio de 2011) y Resolución de 6 de octubre de 2005, de la Dirección General de Gestión de Recursos Humanos, por la que se aprueba el Manual para la gestión del cumplimiento de la jornada y horarios en los Centros Públicos de Educación y Servicios Educativos dependientes de la Consejería de Educación de la Junta de Andalucía (Boletín Oficial de la Junta de Andalucía, número 204, de 19 de octubre de 2005).
} 
forma aleatoria, anónima y voluntaria. De esta forma, se obtuvo una muestra de 1,276 participantes. Al cuestionario se le aplicó un análisis de la consistencia mediante el cálculo del alfa de Cronbach de forma global, para cada dimensión y, por último, de forma individual, a cada ítem, analizando la variación producida en el resultado global del test y en el de cada dimensión, al eliminar cada uno de estos.

Por último, se analizó la validez del test mediante un análisis factorial en el que se utilizó el método de componentes principales, debido al carácter ordinal de las variables utilizadas, y usando para ello la matriz de covarianzas, por la homogeneidad de escala de las variables incluidas en el estudio. Para la extracción de factores se utilizó la regla de Kaiser. Sobre el resultado se aplicó la rotación Varimax de los ejes con el objetivo de minimizar el número de variables que tienen saturaciones altas en cada factor, lo que simplifica y ayuda a la interpretación de los factores o dimensiones estudiadas.

\section{Resultados}

El estudio contó con una muestra de 1,276 participantes ( $77.8 \%$ de madres y $22.2 \%$ de padres). El $64.7 \%$ de ellos tenía una edad comprendida entre los 35 y los 44 años, y el $60.4 \%$ contaban con dos hijos. Un $44.4 \%$ de los encuestados tenía un hijo matriculado en el centro, mientras que un $46.8 \%$ tenía dos. El 97.7\% tenía un hijo en educación primaria, un $21.9 \%$ en educación infantil y un $16.3 \%$ en Educación Secundaria Obligatoria (ESO), y sólo el 3.1\% tenía hijos escolarizados en Bachillerato.

Por otro lado, los dos niveles de estudio predominantes entre los progenitores fueron el universitario, con un $35 \%$, y sólo estudios primarios con un $17.4 \%$.

En relación con la ocupación de los progenitores, el 21.6\% engloba a técnicos, profesionales, científicos o intelectuales; el $17.2 \%$ se dedica a las tareas domésticas y un $13.1 \%$ a trabajos sin cualificación específica.

Se realizó un análisis descriptivo previo de los ítems mediante el cálculo de la media y la desviación estándar, que tenía como objetivo obtener valoraciones preliminares de las respuestas, debido a su carácter ordinal. Las puntuaciones medias oscilaron entre 1.30 y 4.56; el rango de puntaciones de las desviaciones típicas fluctuó entre 0.883 y 1.659.

Los ítems 16, 17, 18 y 19 hacen referencia al medio a través del cual el tutor cita a los padres (teléfono, cartas, agenda y medios telemáticos). El carácter excluyente de sus respuestas recomienda su eliminación del análisis de la consistencia, por su bajo resultado.

El alfa de Cronbach global del cuestionario (0.953) confirman su consistencia. Éste, además, no mejoró si se eliminaba cualquier ítem, por lo que todos los ítems resultaron relevantes. Al analizar la consistencia de cada dimensión por separado se obtuvieron valores comprendidos entre 0.724 y 0.927 , resultados que se muestran en la tabla I.

Tabla I. Fiabilidad de las dimensiones de la escala

\begin{tabular}{l|l|c|c}
\hline Dimensiones & Factor & ítems & $\begin{array}{l}\text { Alfa } \\
\text { de Cronbach }\end{array}$ \\
\hline Recursos y procedimientos utilizados para la comunicación & $8 / 10 / 11 / 13$ & 10 & .724 \\
entre la escuela y la familia. & 3 & 8 & .890 \\
Contenidos de las comunicaciones con las familias. & 1 & 12 & .927 \\
Conocimiento de las familias sobre aspectos del centro. & 7 & .822 \\
Participación de las familias en el centro, que incluía la participación & $7 / 9$ & 7 & .870 \\
en actividades del centro y en estructuras organizativas del mismo. & & 17 & .923 \\
Contribución de las familias en el aprendizaje de sus hijos e hijas. & 4 & 5 & .817 \\
Facilidades para la colaboración/participación en el centro escolar. & $2 / 5 / 12$ & 6 & \\
Compromisos de mejora por parte de las familias. & 6 & & \\
\hline
\end{tabular}

Рara determinar la validez del constructo y, por tanto, la idoneidad de la aplicación del análisis factorial, se halló la medida de adecuación muestral de Kaiser-Meyer-Olkin (KMO=0.950) para las 62 variables de la 
escala definitiva y la prueba de esfericidad de Bartlett ( $p=0.000)$ con todas las correlaciones significativas. Además, la matriz anti-imagen contiene valores altos para la diagonal y cercanos a 0 en el resto, y para el determinante de la matriz de correlaciones se obtuvo un valor de 1,779 y para la matriz de covarianzas 0.001 .

El Análisis Factorial Exploratorio (AFE) comenzó con la obtención de comunalidades, obtenidas mediante el análisis de componentes principales para toda la escala, siendo los valores más bajos 0.357 (ítem 15) y 0.460 (ítem 13). Tras estos, el resto oscila entre 0.5 (ítem 29) y 0.853 (ítem 78). La varianza explicada por los 12 factores extraídos por la regla de Kaiser fue de 63.901\% (por encima del límite establecido por Henson y Roberts, 2006). Se llevó a cabo una rotación de los factores por el método Varimax para una mejor interpretación de los resultados.

La dimensión "recursos utilizados para la comunicación entre la escuela y la familia", se corresponde con el factor 8 configurado en el AFE, diferenciándose entre ambos modelos en 2 ítems, que definen los factores 11 y 12 .

Las dimensiones "contenidos de las comunicaciones con las familias" y "conocimiento de las familias sobre aspectos del centro", establecidas a nivel teórico, coinciden íntegramente con los factores 4 y 10 del AFE, respectivamente.

La quinta dimensión, "participación de las familias en el centro", incluía la participación en actividades del centro y en estructuras organizativas del mismo hecho que ha quedado reflejado en los factores 7 y 9 del AFE.

La dimensión "contribución de las familias en el aprendizaje de sus hijos", diseñada a nivel teórico, también se puede apreciar recogida íntegramente en el factor 3 del AFE.

La séptima dimensión, "Facilidades para la colaboración/participación en el centro escolar", se ha visto distribuida en tres factores en el AFE. El primero de ellos (factor 2) hace referencia a aspectos generales de la colaboración familia y escuela, el segundo (factor 5) se corresponde con iniciativas del centro que fomentan la colaboración o participación familiar, mientras que el tercero (factor 8) gira en torno a la implicación personal de las familias en esta colaboración.

Por último, la dimensión "compromisos de mejora por parte de las familias" se corresponde con el factor 6 del AFE.

La tabla II muestra los resultados de las comunalidades de cada ítem y el valor del alfa de Cronbach del instrumento al eliminar cada uno de ellos. 
Tabla II. Comunalidades y alfa de Cronbach

\begin{tabular}{|c|c|c|c|}
\hline Dimensión & ítem & Comunalidades & $\begin{array}{l}\text { Alfa de Cronbach } \\
\text { al eliminarlo }\end{array}$ \\
\hline \multirow{6}{*}{$\begin{array}{l}\text { 1. Recursos y procedimientos utilizados para la } \\
\text { comunicación entre la escuela } \\
\text { y la familia. }\end{array}$} & 10 & .572 & .952 \\
\hline & 11 & .575 & .952 \\
\hline & 12 & .720 & .952 \\
\hline & 13 & .460 & .952 \\
\hline & 14 & .836 & .953 \\
\hline & 15 & .357 & .953 \\
\hline \multirow{8}{*}{$\begin{array}{l}\text { 2. Contenidos de las comunicaciones } \\
\text { con las familias. }\end{array}$} & 20 & .756 & .952 \\
\hline & 21 & .675 & .952 \\
\hline & 22 & .617 & .952 \\
\hline & 23 & .622 & .952 \\
\hline & 24 & .597 & .952 \\
\hline & 25 & .725 & .952 \\
\hline & 26 & .791 & .952 \\
\hline & 27 & .741 & .952 \\
\hline \multirow{12}{*}{$\begin{array}{l}\text { 3. Conocimiento de las familias } \\
\text { sobre aspectos del centro. }\end{array}$} & 28 & .578 & .952 \\
\hline & 29 & .500 & .952 \\
\hline & 30 & .536 & .952 \\
\hline & 31 & .724 & .951 \\
\hline & 32 & .728 & .951 \\
\hline & 33 & .711 & 951 \\
\hline & 34 & .619 & .952 \\
\hline & 35 & .578 & .952 \\
\hline & 36 & .588 & .951 \\
\hline & 37 & .591 & .951 \\
\hline & 38 & .569 & .952 \\
\hline & 39 & .603 & .952 \\
\hline \multirow{7}{*}{$\begin{array}{l}\text { 4. Participación de las familias en el centro, } \\
\text { que incluía la participación en actividades del } \\
\text { centro y en estructuras organizativas del } \\
\text { mismo. }\end{array}$} & 40 & .603 & .952 \\
\hline & 41 & .669 & .952 \\
\hline & 42 & .599 & .952 \\
\hline & 43 & .541 & .952 \\
\hline & 44 & .650 & .952 \\
\hline & 48 & .641 & .952 \\
\hline & 49 & .568 & .953 \\
\hline \multirow{7}{*}{$\begin{array}{l}\text { 5. Contribución de las familias en el } \\
\text { aprendizaje de sus hijos. }\end{array}$} & 50 & .568 & .952 \\
\hline & 51 & .534 & .952 \\
\hline & 52 & .617 & .952 \\
\hline & 53 & .638 & .952 \\
\hline & 54 & .615 & .952 \\
\hline & 55 & .514 & .952 \\
\hline & 56 & .521 & .952 \\
\hline \multirow{17}{*}{$\begin{array}{l}\text { 6. Facilidades para la colaboración/ } \\
\text { participación en el centro escolar. }\end{array}$} & 57 & .538 & .952 \\
\hline & 58 & .565 & .952 \\
\hline & 59 & .564 & .952 \\
\hline & 60 & .567 & .952 \\
\hline & 61 & .566 & .952 \\
\hline & 62 & .644 & .952 \\
\hline & 63 & .643 & .951 \\
\hline & 64 & .651 & .952 \\
\hline & 65 & .619 & .952 \\
\hline & 66 & .634 & .951 \\
\hline & 67 & .580 & .952 \\
\hline & 68 & .597 & .952 \\
\hline & 69 & .597 & .952 \\
\hline & 70 & .647 & .952 \\
\hline & 71 & .715 & .952 \\
\hline & 72 & .732 & .952 \\
\hline & 73 & .641 & .952 \\
\hline \multirow{5}{*}{$\begin{array}{l}\text { 7. Compromisos de mejora por parte } \\
\text { de las familias }\end{array}$} & 74 & .692 & .953 \\
\hline & 75 & .760 & .952 \\
\hline & 76 & .706 & .953 \\
\hline & 77 & .634 & .952 \\
\hline & 78 & .853 & .953 \\
\hline
\end{tabular}




\section{Discusión y conclusiones}

Aunque las relaciones familia y escuela han sido definidas y analizadas desde diferentes perspectivas y en menor o mayor grado de profundidad, existen pocos instrumentos que permitan un conocimiento exhaustivo de estas relaciones (Valdés, Carlos y Arreola, 2013). La Escala de Diagnóstico de las Relaciones Familia-Escuela (DIREFAES) pretende ofrecer una herramienta para seguir avanzando en el conocimiento de ambas instituciones y las relaciones que se establecen entre ellas. Sus características psicométricas posibilitan su uso en el diagnóstico de estas relaciones. No obstante, el instrumento requiere ser depurado, tal y como se ha realizado con los ítems 16,17, 18 y 19, de este modo se conseguirá fortalecer las propiedades psicométricas del mismo, ya que el AfE ha revelado una desconexión entre algunas dimensiones teóricas y los factores surgidos.

A la vista de este análisis psicométrico, la propuesta definitiva de dimensiones e ítems se puede apreciar en el Anexo 1, siendo éste un primer paso para obtener información valiosa sobre las relaciones familiaescuela, especialmente en aspectos como el conocimiento de las familias sobre el centro, el contenido de las comunicaciones, la contribución de las familias en el aprendizaje de sus hijos y los compromisos de mejora por parte de las familias. En definitiva, el instrumento constituye una nueva herramienta para conseguir un conocimiento más exhaustivo de las relaciones que se establecen entre estos dos espacios de desarrollo infantil.

\section{Referencias}

American Educational Research Association; American Psychological Association y National Council for Measurement in Education. (1999). Standards for educational and psychological tests. Washington, DC: AERA.

Backhoff, E., Bouzas, A., González, M., Andrade, E., Hernández, R. y Contreras, C. (2008). Factores asociados al aprendizaje de estudiantes de 3o. de Primaria en México. Recuperado de http://www.senado.gob.mx/comisiones/educacion/Escuela Libre Violencia/docs/Factoresescolarestercer o2008.pdf

Bodovski, K. y Youn, M. (2010). Love, discipline, and elementary school achievement: the role of family emotional climate. Social Science Research, 39(4), 585-595.

Bolívar, A. (2006). Familia y escuela: dos mundos llamados a trabajar en común. Revista de Educación, 339, 119-146. Recuperado de http://www.revistaeducacion.mec.es/re339/re339a08.pdf

Carretero-Dios, H. y Pérez, C. (2005). Normas para el desarrollo y revisión de estudios instrumentales. International Journal of Clinical and Health Psychology, 5(3), 521-551.

Chiu, M. y Xihua, Z. (2008). Family and motivation effects on mathematics achievement: analyses of students in 41 countries. Learning and Instruction, 18(4), 321-336.

Colás, P. y Contreras, J. A. (2013). La participación de las familias en los centros de Educación Primaria. Revista de Investigación Educativa, 31(2), 485-499. doi:10.6018/rie.31.2.171031

Córdoba, L., García, V., Luengo, L., Vizuete, M. y Feu, S. (2011). Determinantes socioculturales: su relación con el rendimiento académico en alumnos de Enseñanza Secundaria Obligatoria. Revista de Investigación Educativa, 29(1), 83-96. Recuperado de http://revistas.um.es/rie/article/view/110361

Dearing, E., Kreider, S. y Weiss, H. (2006). Family involvement in school and low-income children's literacy performance: longitudinal associations between and within families. Journal of Educational Psychology, 98(4), 653-664. 
Domingo, J., Martos, M. y Domingo, L. (2010). Colaboración familia-escuela en España: retos y realidades. REXE. Revista de Estudios y Experiencias en Educación, 9(18), 111-133.

Driessen, G., Smit, F. y Sleegers, P. (2005). Parental involvement and educational achievement. British Educational Research Journal, 31(4), 509-532.

Fan, W., Williams, C. y Wolters, C. (2011). Parental involvement in predicting school motivation: similar and differential effects across ethnic groups. The Journal of Educational Research, 105(1), 21-35.

Fraguela, R., Lorenzo, J. J. y Varela, L. (2011). Escuela, familia y ocio en la conciliación de los tiempos cotidianos de la infancia. Revista de Investigación Educativa, 29(2), 429-446.

Gamarra, C. y Carrasco, A. (2013). Relación entre padres-escuela a través de la utilización del facebook como medio de comunicación. Revista de Investigación en Psicología, 16(1), 45-73.

García, M. P., Gomariz, M. A., Hernández, M. A. y Parra, J. (2010). La comunicación entre la familia y el centro educativo, desde la percepción de los padres y madres de los alumnos. Educatio Siglo XXI, 28(1), 157-187.

Hambleton, R., Swaminathan, H. y Rogers, H. (1991). Fundamentals of Item Response Theory (Vol. 2). Londres: Sage.

Henson, R. y Roberts, K. (2006). Use of exploratory factor analysis in published research: common errors and some comment on improved practice. Educational and Psychological Measurement, 66(3), 393-416.

Lord, F. (1980). Applications of item response theory to practical testing problems. Hillsdale, NJ: Lawrence Erlbaum Associates.

Lozano, J., Alcaraz, S. y Colás, P. (2013). Los centros educativos multiculturales y sus relaciones con las familias: el caso de la región de Murcia. Revista Educación XX1, 16(1), 210-232.

Martínez-Arias, R. (1995). Psicometría: teoría de los test psicológicos y educativos. Madrid: Síntesis.

Muñiz, J. (1997). Introducción a la Teoría de Respuesta de los ítems. Madrid: Pirámide.

Muñiz, J. (1998). Teoría clásica de los test. Madrid: Pirámide.

Navarro, E., Expósito, E., López, E. y Thoilliez, B. (2014). EPIBI: Escala de Percepción de Indicadores de Bienestar Infantil. Validación del instrumento utilizando modelos politómicos de Rash. Revista de Educación, 364, 39-65. doi:10.4438/1988-592X-RE-2014-364-254

OECD (2012). Let's read them a story! The parent factor in education. Recuperado de https://www.oecd.org/pisa/pisaproducts/Parent\%20Factor e-booknew\%20logo FINAL new\%20page\%2047.pdf

Pozo, M. T., Gutiérrez, J. y Rodríguez, C. (2007). El uso del método Delphi en la definición de los criterios para una formación de calidad en animación sociocultural y tiempo libre. Revista de Investigación Educativa, 25(2), 351-366. Recuperado de http://revistas.um.es/rie/article/view/96831

Rodríguez, A., Droguett, L. y Revuelta, L. (2012). School and personal adjustment in adolescence: the role of academic self-concept and perceived social support. Revista de Psicodidáctica, 17(2), 397-414.

Ruiz de Miguel, C. (2009). Las escuelas eficaces: Un estudio multinivel de factores explicativos del rendimiento escolar en el área de matemáticas. Revista de Educación, 348, 355-376. 
Santos, M. A., Lorenzo M. y Priegue, D. (2011). Infancia de la inmigración y educación: la visión de las familias. Revista de Investigación Educativa, 29(1), 97-110.

Sarramona, J. y Roca, E. (2007). La participación de las familias en la escuela como factor de calidad educativa. Participación Educativa, 4, 25-33.

Valdés, A., Carlos, E. y Arreola, C. (2013). Desarrollo de un instrumento para medir la participación de los padres en la educación escolar de los hijos. Revista de Evaluación Educativa, 2(1). Recuperado de http://revalue.mx/revista/index.php/revalue/issue/view/4

Van der Linden, W. y Hambleton, R. (1997). Handbook of modern item response theory. Nueva York: Springer.

Villiger, C., Niggli, A. y Wandeler, C. (2011). Does family make a difference? Mid-term effects of a school home based intervention program to enhance reading motivation. Learning and Instruction, 22(2), 79-91. 


\section{Anexo 1}

Anexo 1. Dimensiones e ítems propuesto para la escala DIREFAES

\begin{tabular}{|c|c|}
\hline Dimensiones & ítems \\
\hline $\begin{array}{l}\text { Recursos y procedimientos } \\
\text { utilizados para la } \\
\text { comunicación entre la escuela } \\
\text { y la familia }\end{array}$ & $\begin{array}{l}\text { 10. Horario de tutoría. } \\
\text { 11. Reuniones grupales con tutor. } \\
\text { 12. Entrevistas personales con el tutor. } \\
\text { 13. Contacto casual con el tutor. } \\
\text { 14. Agenda escolar. } \\
\text { 15. Tutoría electrónica. }\end{array}$ \\
\hline $\begin{array}{l}\text { Contenidos de las } \\
\text { comunicaciones con las } \\
\text { familias }\end{array}$ & $\begin{array}{l}\text { 20. Disciplina o la asistencia a clase. } \\
\text { 21. Actividades extraescolares del centro. } \\
\text { 22. Aspectos positivos del aprendizaje de los hijos. } \\
\text { 23. Aspectos negativos y/o dificultades de aprendizaje de sus hijos. } \\
\text { 24. Materiales, libros, uniforme o aspectos organizativos. } \\
\text { 25. Aspectos relacionados con el desarrollo personal de los hijos/as, sus } \\
\text { capacidades, gustos, etc. } \\
\text { 26. Grado de adaptación al aula y/o relación con compañeros. } \\
\text { 27. Colaboración que se presta en casa al proceso educativo. }\end{array}$ \\
\hline $\begin{array}{l}\text { Conocimiento de las familias } \\
\text { sobre aspectos del centro }\end{array}$ & $\begin{array}{l}\text { 28. Estructura y funcionamiento del centro. } \\
\text { 29. Tutor o tutora. } \\
\text { 30. Resto del profesorado. } \\
\text { 31. Proyecto Educativo de Centro. } \\
\text { 32. Reglamento de Organización y Funcionamiento. } \\
\text { 33. Plan de Orientación y Acción Tutorial. } \\
\text { 34. Plan de Convivencia (normas, sanciones, estructuras de } \\
\text { convivencia...). } \\
\text { 35. Programación de Aula (incluidos criterios y procedimientos de } \\
\text { evaluación, medidas de apoyo y refuerzo...). } \\
\text { 36. Horario de atención a las familias. } \\
\text { 37. Derechos y deberes de las familias. } \\
\text { 38. Actividades del Ampa. } \\
\text { 39. Delegado/a de madres y padres de curso y Junta de Delegados/as. }\end{array}$ \\
\hline $\begin{array}{l}\text { Participación de las familias } \\
\text { en el centro, que incluía la } \\
\text { participación en actividades } \\
\text { del centro y en estructuras } \\
\text { organizativas del mismo }\end{array}$ & $\begin{array}{l}\text { 40. Partcipación en actividades en la casa. } \\
\text { 41. Participación en actividades complementarias. } \\
\text { 42. Participación en actividades extraescolares. } \\
\text { 43. Asistencia a charlas, talleres de formación. } \\
\text { 44. Participación en otras actividades del centro. } \\
\text { 48. Participación activa en el AmpA. } \\
\text { 49. Participación en reuniones de delegados de familias. }\end{array}$ \\
\hline $\begin{array}{l}\text { Contribución de las familias } \\
\text { en el aprendizaje de sus hijos } \\
\text { e hijas }\end{array}$ & $\begin{array}{l}\text { 50. Hablo con mi hijo/a sobre sus estudios. } \\
\text { 51. Superviso y controlo sus tareas escolares. } \\
\text { 52. Le ayudo en la organización de su tiempo de estudio. } \\
\text { 53. Fomento la realización de actividades culturales en la familia. } \\
\text { 54. Me informo de actividades de ocio para mi hijo/a. } \\
\text { 55. Apoyo la asistencia de mi hijo a actividades complementarias como } \\
\text { excursiones, celebración de festividades, etc. } \\
\text { 56. Procuro en casa un ambiente de trabajo adecuado (libre de ruidos y } \\
\text { distracciones, con los materiales adecuados...). }\end{array}$ \\
\hline
\end{tabular}


Facilidades para la colaboración/participación en el centro escolar
57. Asistencia al centro por iniciativa propia para hablar con el tutor. 58. Búsqueda de disponibilidad horaria para asistir y colaborar con el centro.

59. El tutor facilita que las familias se sientan cómodas.

60. El colegio facilita la asistencia a las tutorías y reuniones con el horario establecido.

61. El profesorado y familias tienen los mismos objetivos educativos

62. Confianza del tutor para hablar de los hijos.

63. El centro ofrece información fluida y suficiente.

64. En el colegio hay facilidad para expresar las propias ideas.

65. El colegio admite mejoras educativas propuestas por las familias. 66. En el colegio encuentro apoyos y asesoramiento en temas educativos.

67. El profesorado solicita ayuda y colaboración.

68. En el colegio hay accesibilidad y disposición del profesorado para hablar.

69. El centro promociona actividades en las que participen las familias. 70. Existen cauces para que las familias realicen aportaciones al proyecto educativo del centro.

71. El centro propone actividades de extensión cultural dirigidas a las familias.

72. El colegio propone actividades formativas conjuntas entre profesorado y familias.

73. El centro facilita el acceso a recursos del entorno para las familias. 74. Utilizar más la agenda escolar.

75. Asistir más al centro.

76. Asistir más con otro horario.

77. Apoyar al profesorado en todo.

78. Utilizar el correo electrónico, plataforma PASEN... 